\title{
Respiration-Averaged CT for Attenuation Correction in Canine Cardiac PET/CT
}

\author{
Russell A.H. Cook ${ }^{1,2}$, Greg Carnes ${ }^{1,3}$, Ting-Yim Lee ${ }^{1-4}$, and R. Glenn Wells ${ }^{1,2,4}$ \\ ${ }^{I}$ Department of Medical Biophysics, University of Western Ontario, London, Ontario, Canada; ${ }^{2}$ Imaging Program, Lawson Health \\ Research Institute, London, Ontario, Canada; ${ }^{3}$ Imaging Research Laboratories, Robarts Research Institute, London, Ontario, Canada; \\ and ${ }^{4}$ Nuclear Medicine, St. Joseph's Health Care London, London, Ontario, Canada
}

Heart disease is a leading cause of death in North America. With the increased availability of PET/CT scanners, CT is now commonly used as a transmission source for attenuation correction. Because of the differences in scan duration between PET and CT, respiration-induced motion can create inconsistencies between the PET and CT data and lead to incorrect attenuation correction and, thus, artifacts in the final reconstructed PET images. This study compared respiration-averaged CT and 4-dimensional (4D) CT for attenuation correction of cardiac PET in an in vivo canine model as a means of removing these inconsistencies. Methods: Five dogs underwent respiration-gated cardiac ${ }^{18} \mathrm{~F}-\mathrm{FDG}$ PET and 4D CT. The PET data were reconstructed with 3 methods of attenuation correction that differed only in the CT data used: The first method was single-phase CT at either end-expiration, end-inspiration, or the middle of a breathing cycle; the second was respiration-averaged CT, which is CT temporally averaged over the entire respiratory cycle; and the third was phase-matched CT, in which each PET phase is corrected with the matched phase from 4D CT. After reconstruction, the gated PET images were summed to produce an ungated image. Polar plots of the PET heart images were generated, and percentage differences were calculated with respect to the phase-matched correction for each dog. The difference maps were then averaged over the 5 dogs. Results: For single-phase CT correction at end-expiration, end-inspiration, and mid cycle, the maximum percentage differences were $11 \% \pm 4 \%, 7 \% \pm$ $3 \%$, and $5 \% \pm 2 \%$, respectively. Conversely, the maximum difference for attenuation correction with respiration-averaged CT data was only $1.6 \% \pm 0.7 \%$. Conclusion: Respirationaveraged CT correction produced a maximum percentage difference 7 times smaller than that obtained with end-expiration single-phase correction. This finding indicates that using respiration-averaged CT may accurately correct for attenuation on respiration-ungated cardiac PET.

Key Words: PET; 4D CT; attenuation correction; respirationinduced motion; cardiac imaging

J Nucl Med 2007; 48:811-818

DOI: 10.2967/jnumed.106.034199

Received Jul. 3, 2006; revision accepted Oct. 31, 2006.

For correspondence or reprints contact: R. Glenn Wells, PhD, Lawson Health Research Institute, Nuclear Medicine Department, Room C531, St. Joseph's Hospital, 268 Grosvenor St., London, Ontario, Canada, N6A 4V2.

E-mail: wells@lawsonimaging.ca

COPYRIGHT @ 2007 by the Society of Nuclear Medicine, Inc.
C ardiovascular disease causes 1 in 3 deaths in Canada (1) and worldwide (2) every year. The largest component of cardiovascular disease is heart disease. Proper patient management after a heart attack is crucial to maximize recovery. Cardiac imaging of ${ }^{18}$ F-FDG uptake with PET, currently the gold standard for determining the viability of heart tissue, allows the clinician to determine whether the tissue is ischemic or necrotic (3). This distinction is a clinical factor guiding the clinician toward treating the patient medically, with bypass surgery, or with heart transplantation (4).

Accurate PET images require attenuation correction for signal loss via photon absorption in patient tissues (5). This correction is done through the use of a transmission scan. In the past, the transmission image, or map, has generally been acquired through the use of a radioisotope source such as ${ }^{68} \mathrm{Ge}$. However, the low $511-\mathrm{keV}$ photon flux generated by such radioisotopes requires lengthy transmission scans. Because the length of a radioisotope transmission scan is similar to that of a PET emission scan, the 2 data sets are consistent and the problem of respiratory motion is reduced, leading it to have received minimal attention (6) despite having been recognized for some time (7).

With the advent of hybrid PET/CT scanners, the use of CT for attenuation correction has become common (8), and in some of these hybrid scanners, radioisotope attenuation correction is no longer available. Some advantages of using CT as a transmission source for attenuation correction include its speed, nearly noise-free images, and high spatial resolution. These advantages increase patient throughput, improve the signal-to-noise ratio of the attenuation-corrected PET images, and accurately localize tracer uptake anatomically. However, the speed of the CT scan is also a potential source of problems. The typical temporal resolution of a CT image is less than $1 \mathrm{~s}$, significantly shorter than the 4-s resting breathing period of a person (9). CT therefore acquires a snapshot image of the patient at a single phase of the respiratory cycle. A cardiac PET scan requires 15-30 min per acquisition and gives an image that is temporally averaged over many respiratory cycles. Inconsistencies between a $\mathrm{CT}$ attenuation correction map from a single 
respiratory phase and a temporally averaged PET study imaged over many breathing periods may lead to errors in the final attenuation-corrected image (10). This respirationinduced misregistration between the PET and CT scans has brought about increased interest in methods to correct for artifacts from respiratory motion $(6,10-16)$.

Respiration causes the heart to move by up to $1.6 \mathrm{~cm}$, which is about the thickness of the heart wall (13). In other words, the entire heart wall can move into the adjacent lung region, and that part of the heart will consequently be undercorrected. Errors resulting from the PET/CT data mismatch can seriously affect the count levels in the reconstructed heart image (14). Studies of simulations (10), canines (14), and humans (15) have shown that attenuation correction based on CT scans acquired at a single phase of the respiratory cycle, such as at end-expiration or end-inspiration, leads to incorrect calculated levels of heart activity. Additionally, these errors are also seen clinically in ${ }^{18} \mathrm{~F}$-FDG PET studies corrected with a standard helical CT scan $(16,17)$, possibly leading to a change in diagnosis or treatment of the patient (18).

One solution to this temporal mismatch is to reduce the influence of respiration-induced motion on the data through gating of the PET acquisition, wherein the PET data are separated into multiple temporal bins based on the phase of the respiratory cycle (19). Each bin (gate) contains only a small portion of the respiratory cycle and correspondingly reduces the motion present in that gate. The CT information can be organized in a similar fashion by obtaining a 4-dimensional (4D) CT dataset (9). The reconstructed CT images are retrospectively reordered to produce $\mathrm{CT}$ volumes distributed throughout the respiratory cycle, with each phase having approximately the same temporal duration as the PET gates. Each individual PET gate and the reordered 4D CT can be matched for respiratory phase, allowing consistent attenuation correction of all PET gates throughout the respiratory cycle. The problem with this phase-matched approach is that it is both computationally and procedurally complex, because the 4D CT images have to be separately reconstructed for each individual respiratory phase and then matched to the gated PET data for image reconstruction with attenuation correction. This approach is potentially difficult to implement clinically. Furthermore, routine clinical cardiac studies are not respiration-gated.

A simpler solution to the temporal misregistration problem is to obtain a CT volume temporally averaged over the respiratory cycle of the patient, or a respiration-averaged CT volume. Such a CT volume has the same temporal properties as the respiration-ungated PET data, and the approach has shown promise in oncology (17). This solution is more easily implemented than the phase-matched technique, because matching of the PET and CT phases is not required, nor is triggering of either acquisition at a particular phase of the respiratory cycle.

In this study, we examined the effects of respiration in a canine model of cardiac ${ }^{18}$ F-FDG PET. The objective of our study was to compare the accuracy of respiration-averaged CT with phase-matched CT for attenuation correction of respiration-ungated PET images.

\section{MATERIALS AND METHODS}

Five dogs, weighing approximately $22 \mathrm{~kg}$ each, were anesthetized with a 15 - to $20-\mathrm{mL}$ dose of $1 \%$ propofol and maintained with $1.5 \%-2 \%$ isoflurane gas. This study was approved by the Animal Use Subcommittee of the Canadian Council on Animal Care at the University of Western Ontario (ethics approval 2005002-02). The dogs were intubated and ventilated for the duration of the study, with the ventilation rate set at 8 breaths per minute and a peak inspiratory pressure of $16 \mathrm{~cm}$ of $\mathrm{H}_{2} \mathrm{O}$. The resultant respiratory cycle of the dogs was asymmetric, with inspiration taking place over the first third of the cycle and expiration encompassing the remaining two thirds. The heart rate was monitored throughout and had a mean value of 103.6 beats per minute for the 5 animals, with a range of 91-115. The dogs were laid supine, to mimic human positioning during clinical examinations, and injected with $260 \mathrm{MBq}$ of ${ }^{18} \mathrm{~F}-\mathrm{FDG}$.

Immediately after tracer injection, a 4D CT cine transmission scan was acquired with $\mathrm{CT}$ parameters set to the default values for a clinical PET/CT examination $(80 \mathrm{~mA} ; 120 \mathrm{kV})$. All scans were acquired on a 4-slice Discovery LS PET/CT scanner (GE Healthcare) at St. Joseph's Health Care London. The total axial field of view was $15 \mathrm{~cm}$ (1 PET bed position), centered on the heart. The 4D CT data were acquired in a shoot (cine)-and-step format, with a scan time of $8.2 \mathrm{~s}$ (1-cm field of view) to ensure that a complete dataset for an entire breath cycle $(7.5 \mathrm{~s})$ was acquired at each bed position before moving to the next. However, because no triggering is used during cine CT acquisitions, the initial phase of the respiratory cycle that was captured was different at each bed position. Therefore, to ensure a consistent dataset within each CT volume, we temporally aligned the constituent slices from successive CT bed positions using software that compared the position of the chest wall in abutting transaxial CT images. This approach is similar to that of Pan et al. (9), who use internal measurements and do not require a respiratory triggering device to align successive CT bed positions. The rotation speed of the CT gantry was $500 \mathrm{~ms}$ per revolution, and projection data from a full $360^{\circ}$ were used for image reconstruction. In other words, each reconstructed image corresponded to a temporal width of $500 \mathrm{~ms}$ of the respiratory cycle and was equivalent to a bin in the respiration-gated PET acquisition as described below. From an 8.2-s cine scan at each bed position, 78 images were reconstructed by offsetting the 500-ms temporal width of each successive image by $100 \mathrm{~ms}$. From these 78 images, 75 images corresponding to 1 breath cycle, for each bed position, were aligned in respiratory phase with respect to one another. Therefore, 75 3-dimensional CT volumes were produced, offset from one another by $100 \mathrm{~ms}$ (Figure 1A). The $75 \mathrm{CT}$ volumes were then reordered into 5 sets of 15 sequential volumes (Fig. 1B). Within each set of 15 sequential volumes, each volume covered $0.5 \mathrm{~s}$ of the respiratory cycle such that the entire cycle was covered. Each of the 5 sets of 15 volumes was phase-shifted from the other sets by $100 \mathrm{~ms}$. The CT acquisition produced 2.5-mm-thick contiguous slices. The CT volumes were then interpolated to 4.25 -mm-thick contiguous slices to match the PET slice thickness before conversion of the CT image to an attenuation map. 


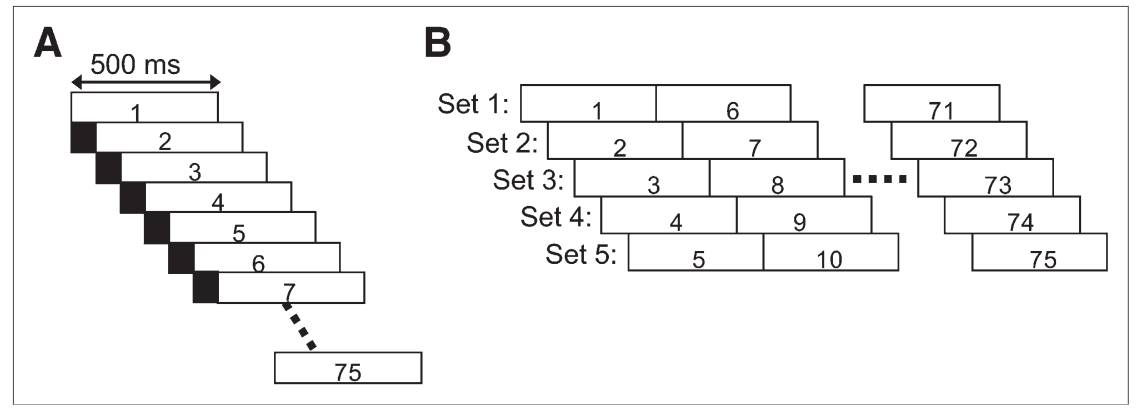

FIGURE 1. Illustration of temporal sampling of 4D CT volumes. (A) Set of 75 volumes each $500 \mathrm{~ms}$ in width and offset from each other by $100 \mathrm{~ms}$ (black boxes). Dashed line illustrates how sixth volume temporally follows first CT volume. (B) Five sets of 15 sequential volumes each encompassing an entire respiratory cycle and phase shifted from one another by $100 \mathrm{~ms}$.

One hour after injection of ${ }^{18} \mathrm{~F}-\mathrm{FDG}$, a half-hour respirationgated PET emission scan was acquired. The PET scan was gated into 15 frames (bins) of $500 \mathrm{~ms}$, using a trigger signal obtained from the end-tidal $\mathrm{CO}_{2}$ concentration in the endotracheal tube connected to the ventilator. The PET data were reconstructed using orderedsubsets expectation maximization, with the clinical default parameters (28 subsets, 2 iterations, and a 2.73-mm 2-dimensional postreconstruction gaussian filter) for the Discovery LS PET/CT scanner. The 15 respiration-gated PET images were then summed to generate an ungated PET image. All results presented in this paper are based on analysis of respiration-ungated PET images, because clinical cardiac PET images are not gated for respiration.

Three different attenuation correction approaches were used during reconstruction of the PET data: single-phase CT, phasematched CT, and respiration-averaged CT.

\section{Single-Phase CT Correction}

The approach with single-phase CT is similar to the current clinical procedure, in which a single high-speed helical CT scan without a breath-hold is used for attenuation correction. In helical CT scans, each transaxial slice is a reconstruction from projection data collected in $0.8 \mathrm{~s}$ and so corresponds to a single phase in the respiratory cycle. Because the patient is breathing while the bed is being translated through the scanner, each slice is acquired at a later phase of the respiratory cycle than the preceding slice. Thus, a clinical CT scan volume comprises a mixture of different single phases. For simplicity, our single-phase correction uses CT volumes in which all transaxial slices are from the same respiratory phase. We have considered CT volumes from 3 different phases for the single-phase attenuation correction: end-expiration, endinspiration, and mid cycle.

\section{Phase-Matched CT Correction}

The phase-matched correction is one in which each respirationgated PET image is attenuation-corrected with the CT image from the same phase of the respiratory cycle. To match the phases of the PET and CT data, we calculated the axial positions of the center of mass of ${ }^{18} \mathrm{~F}-\mathrm{FDG}$ uptake in the heart for the 15 respiration-gated PET images over a respiratory cycle, and we calculated the axial positions of the center of mass of the heart in the corresponding 15 $\mathrm{CT}$ volumes. The bin or gate in which the center-of-mass position of the heart was most inferior corresponded to the bin or gate closest to end-inspiration. The sequence of $15 \mathrm{CT}$ bins was then temporally aligned with the sequence of PET gates based on this near-end-inspiration phase. There are 5 sequences of 4D CT volumes, each of which spans the entire respiratory cycle and so could be used for gated attenuation correction of the PET data. To find which of these 5 sequences of 4D CT volumes most closely matched the PET data, we compared the axial position of the heart in the PET data and in the 4D CT data using the $\chi^{2}$ test. The 4D CT sequence that produced the lowest $\chi^{2}$ value was chosen as the phase-matched set. Phase-matched attenuation correction should be the most accurate, because respiratory motion in each phase is minimal and the PET and CT phases are temporally aligned to minimize discrepancies between the 2 datasets. This phasematched correction is the reference standard to which all other attenuation correction methods were compared.

\section{Respiration-Averaged CT Correction}

To produce a respiration-averaged attenuation correction map, we averaged the sequence of $15 \mathrm{CT}$ volumes from the 4D CT dataset that most closely matched the gated PET data (i.e., the sequence of $15 \mathrm{CT}$ volumes used in the phase-matched correction were averaged). This step generated an attenuation map temporally averaged over the entire breathing cycle in the same way as the respiration-ungated PET data.

For each attenuation correction approach, uptake in the heart was evaluated using polar plot analysis after PET image reconstruction. Percentage difference maps between the phase-matched correction and all other methods of correction, as well as between the end-expiration and end-inspiration corrected images, were created. All percentage difference maps for each dog were calculated with respect to the phase-matched corrected image.

The direction of motion of the heart during respiration varied between animals. We observed that the 2 largest components of heart motion were in the craniocaudal and mediolateral directions. Variations in the ratio of these 2 components led to differences in the direction of motion in the plane orthogonal to the long axis of the heart because in dogs that axis is nearly aligned in the anteroposterior direction. Because we were examining the effects of respiration-induced motion, the reconstructed PET images needed to be aligned with respect to motion and not vascular anatomy. To reduce the effect of differences in the direction of the respiration-induced motion of the heart in different dogs, we calculated motion vectors of the heart in the plane perpendicular to the long axis of the heart for each dog. We determined the motion vectors by measuring the difference between the centroid of the heart at end-expiration and end-inspiration in the apical short-axis slice where respiration-induced motion was largest. This analysis was performed on phase-matched attenuationcorrected PET images because it was the respiration-induced motion of attenuation-corrected PET images that was to be aligned, and phase-matched attenuation correction should provide the most accurate PET images for this purpose. The percentage difference polar maps for the dogs were rotated to align their 
motion vectors to the $1 \mathrm{dog}$ that had a motion vector close to the average of the 5 dogs (Fig. 2). After alignment of the motion vectors, the polar percentage difference maps between the attenuation correction techniques were averaged over the 5 dogs.

Two additional considerations exist with the respirationaveraged CT approach: errors in phase and period matching between the PET and CT data. First, the respiration-averaged CT image was obtained from the sequence of 4D CT volumes phase-matched to the PET data. However, because errors might occur in the matching of phases, the sensitivity of the respiration-averaged CT to possible phase shifts between the PET and CT datasets must be determined. To determine this sensitivity, we averaged the remaining 4 sequences of $15 \mathrm{CT}$ volumes (offset from the matched set by 100, 200, 300 , and $400 \mathrm{~ms}$ ) and used them for attenuation correction. The resulting respiration-averaged attenuation correction maps corresponded to 4 phase shifts separated by $100 \mathrm{~ms}$. Because our respiratory bins were $500 \mathrm{~ms}$, respiration-averaged CT maps from images phase-shifted by $500 \mathrm{~ms}$ or more were equivalent to 1 of the 5 respiration-averaged $\mathrm{CT}$ maps already considered.

Second, a respiration-averaged CT volume obtained over exactly 1 respiratory cycle might not always be obtainable with a 4D CT acquisition in patients, because the axial coverage of the CT scanner is only $2 \mathrm{~cm}$ at most, and scanning at multiple bed positions is needed to cover the entire heart. Therefore, if the patient's breathing period were to change over the course of the 4D CT acquisition, transaxial slices acquired at different bed positions could encompass either more or less than 1 respiratory period and lead to discrepancies in the length of respiration used to generate different portions of the respiration-averaged CT volume. Because the dogs in our study were ventilated, the respiratory period was fixed from one bed position to another, and these discrepancies were not present in our data. We examined these errors by creating incorrectly respiration-averaged CT volumes that contained both $+20 \%$ and $-20 \%$ of the respiratory cycle. Clinically, these errors would be present only in small sections of the 4D CT volume, when the respiratory period fluctuates between bed positions, and would not be present across the entire 4D CT volume. However, our model looked at the worst-case scenario, in which the 4D CT acquisition time at each bed position does not equal the respiratory period, and erred in the same way by either missing or duplicating the same portion of the respiratory cycle. These incorrectly respiration-averaged CT images were achieved by taking 3 consecutive phases including endexpiration, or 3 consecutive phases including end-inspiration, and either doubling or removing their contribution to the respirationaveraged CT. These incorrect respiration-averaged CT volumes were then used for attenuation correction and compared with the images acquired with phase-matched $\mathrm{CT}$ attenuation correction. If these incorrectly respiration-averaged CT volumes did not produce large errors in the PET images when used for attenuation correction, then it is unlikely that the random (uncorrelated) fluctuations in 4D CT acquisitions that may occur clinically will have a large effect.

\section{RESULTS}

The polar plot of ${ }^{18} \mathrm{~F}-\mathrm{FDG}$ uptake in the myocardium, corrected using phase-matched CT and averaged over all 5 subjects, is shown in Figure 3. Also shown in Figure 3 are the averaged difference polar plots for the other methods of attenuation correction. The maximum percentage differences between phase-matched CT attenuation correction and respiration-averaged, end-expiration, endinspiration, and mid cycle CT attenuation corrections were $1.6 \% \pm 0.7 \%, 11 \% \pm 4 \%, 7 \% \pm 3 \%$, and $5 \% \pm 2 \%$, respectively. Finally, the maximum percentage difference between end-expiration and end-inspiration was $17 \% \pm 7 \%$ and represents the maximum possible variation in ${ }^{18} \mathrm{~F}-\mathrm{FDG}$ uptake produced by the use of different phases of the respiratory cycle for attenuation correction.

In individual cases, the errors were larger than those shown in Figure 3, but the spatial distributions of the differences were similar in all cases and so only the average images are presented in this paper. However, the maximum absolute percentage differences from the phase-matched method are shown in a scatter plot in Figure 4 for each individual dog and for each method of CT attenuation correction. Use of a Šidák-corrected paired $t$ test showed that the mean maximum absolute percentage error for respiration-averaged CT attenuation correction with respect to the phase-matched method was significantly $(P<0.05)$ less than those for all other methods of CT attenuation correction.

The results for the 4 phase-shifted respiration-averaged CT attenuation corrections did not visually differ from those for the correct respiration-averaged CT attenuation corrections (Fig. 3B) and are, therefore, not shown. When compared with the correct phase-matched CT attenuation correction image, the maximum percentage differences in ${ }^{18}$ F-FDG uptake were $1.6 \% \pm 0.7 \%$ for the $100-, 300-$, and $400-\mathrm{ms}$ phase shifts and $1.7 \% \pm 0.7 \%$ for the $200-\mathrm{ms}$ phase shift. Figure 5 compares phase-matched and incorrectly respiration-averaged corrections. When the final 3 phases of the cycle, including end-expiration, were included twice in the averaging, the maximum difference was $3 \% \pm 1 \%$. When the 3 consecutive phases, including end-inspiration, were included twice in the averaging, the maximum difference was $1.6 \% \pm 0.6 \%$. When the last 3 phases,
FIGURE 2. Illustration of rotation of polar plots to align respiration-induced motion vectors. (A) Polar plot with respiration-induced motion depicted by arrow. (B) Polar plot with respirationinduced motion depicted by arrow in different direction with respect to A. (C) Polar plot in $B$ rotated such that respirationinduced motion is aligned with that of $A$.

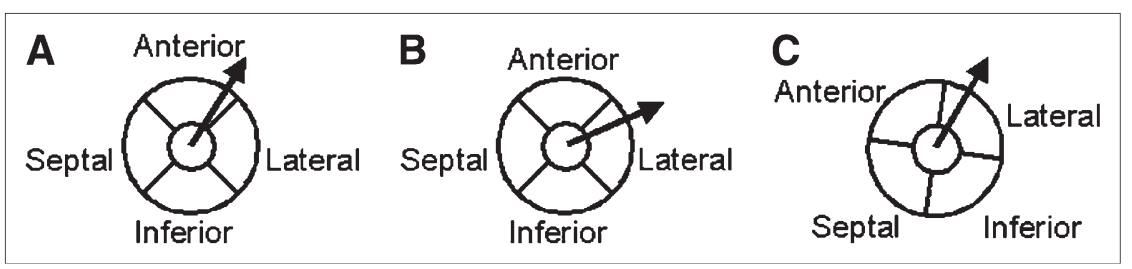




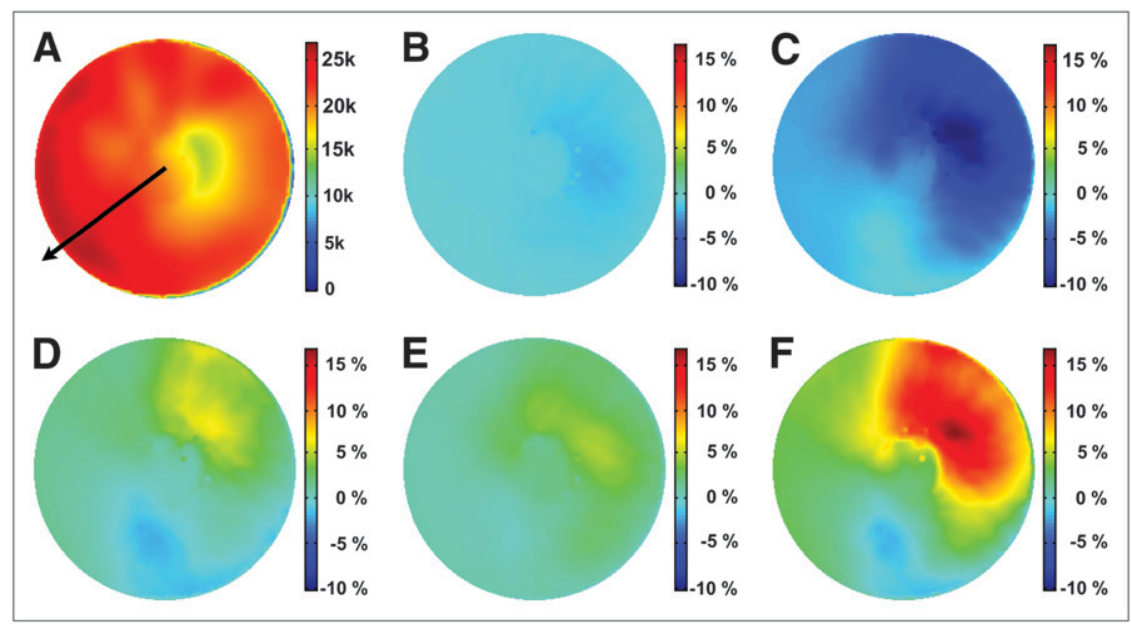

FIGURE 3. Polar plots of ${ }^{18} \mathrm{~F}-\mathrm{FDG}$ uptake in heart and percentage difference in uptake between phase-matched CT attenuation correction and different singlephase attenuation corrections averaged over 5 dogs. (A) Average polar plot with phase-matched CT attenuation correction, with arrow indicating direction of respiration-induced motion to which all polar plots from individual dogs were aligned. (B-E) Polar plots of percentage differences in uptake between phasematched CT attenuation correction and average (B), end-expiration (C), endinspiration (D), and mid cycle (E) CT attenuation corrections. (F) Average polar plot of percentage difference between end-expiration and end-inspiration CT attenuation corrections. All percentage differences were calculated point by point with respect to phase-matched CT attenuation correction of each dog before averaging.

including end-expiration, were omitted from the averaging, the maximum difference was $1.2 \% \pm 0.5 \%$. When 3 consecutive phases, including end-inspiration, were not included in the averaging, the maximum difference was $1.9 \%$ $\pm 0.7 \%$. All percentage difference maps were calculated with respect to phase-matched CT attenuation correction.

Figures 6 and 7 examine the cause of the differences between the phase-matched, end-expiration, and end-inspiration $\mathrm{CT}$ attenuation corrections. Figure 6 shows the $\mathrm{CT}$ image at end-expiration, with the phase-matched-CT-corrected PET image at end-inspiration superimposed in color. A threshold of $50 \%$ of the maximum was applied to the PET data, and the resultant image was superimposed onto the $\mathrm{CT}$ image. Conversely, Figure 7 shows the phase-matched-CT-corrected PET image at end-expiration superimposed on the end-

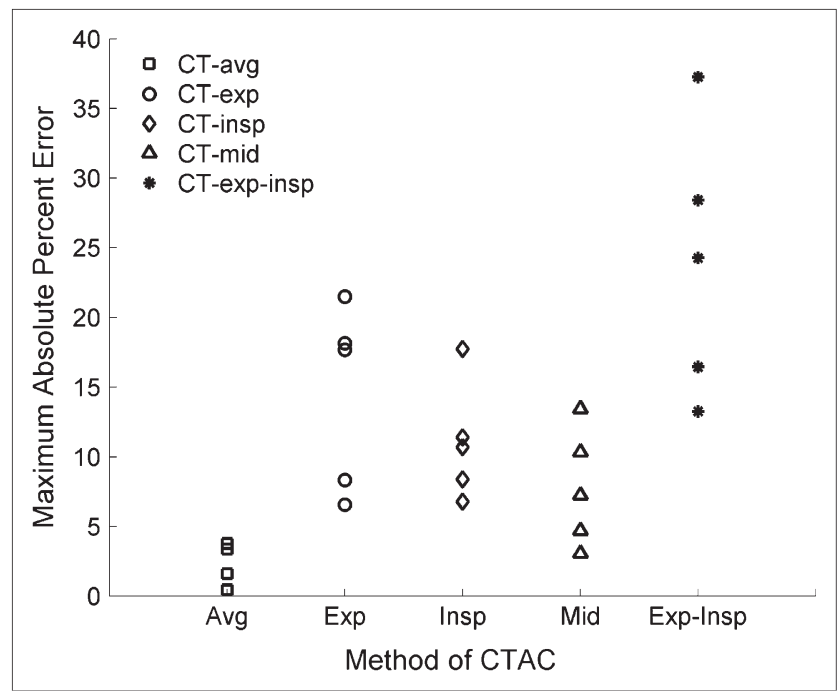

FIGURE 4. Scatter plot showing distribution of maximum absolute errors for all 5 dogs and each method of CT attenuation correction. Errors are expressed as percentage difference from phase-matched CT attenuation correction polar map. inspiration CT image. Figure $6 \mathrm{C}$ clearly demonstrates that the PET image of the heart at end-inspiration extends into the liver region of the end-expiration $\mathrm{CT}$ image. Figures 7A and 7B demonstrate that the PET image of the heart at endexpiration is partly in the lung region of the end-inspiration CT image.

\section{DISCUSSION}

Only the average data of 5 dogs were presented in this paper; however, the results from each individual dog showed

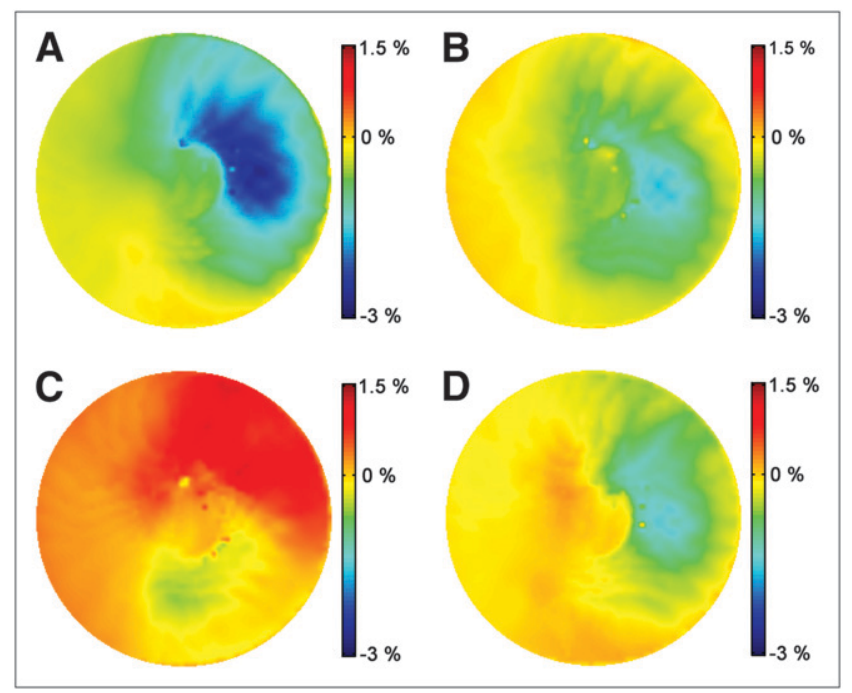

FIGURE 5. Results of attenuation correction with incorrectly respiration-averaged CT. Average, over 5 dogs, of polar plots of percentage differences between phase-matched CT attenuation correction and incorrectly respiration-averaged CT attenuation corrections with final 3 phases of expiration included twice (A), with 3 consecutive phases including end-inspiration included twice $(B)$, with final 3 phases of expiration omitted $(C)$, and with 3 consecutive phases including end-inspiration omitted (D). 
FIGURE 6. Fusion of phase-matched CT attenuation-corrected PET image at end-inspiration with CT image at endexpiration: transaxial slice $(A)$, coronal slice $(B)$, and sagittal slice $(C)$.
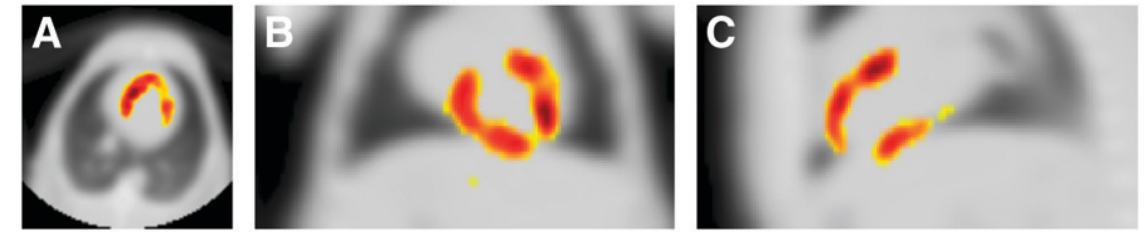

similar relationships between the different methods of attenuation correction. For example, in the dog for which the differences were greatest, the maximum percentage difference between phase-matched correction and end-expiration correction was $20 \%$. For this same dog, the maximum difference between phase-matched and respiration-averaged corrections was $4 \%$, a 5 -fold reduction from the previous single-phase case. This result is similar to what we saw with the average data, because the maximum difference between phase-matched and end-expiration corrections was $11 \% \pm 4 \%$. This difference also dropped by a factor of 7 , to $1.6 \% \pm 0.7 \%$, when phase-matched correction was compared with respiration-averaged correction.

Reorientation of the percentage difference polar plots to align the respiration-induced motion vectors leads to averaging over different anatomic regions of the hearts of the 5 dogs. The fact that the hearts of the 5 dogs were undamaged reduces the heterogeneity of ${ }^{18} \mathrm{~F}-\mathrm{FGD}$ uptake in the heart. Some heterogeneity will remain even in normal hearts, but the effect of these heterogeneities on the average of the difference maps should be minimal, because each difference map is first normalized with respect to its own phasematched-CT-corrected image. However, this approach precludes statements on which anatomic regions of the heart would most likely be affected by respiratory motion. Instead, the effects are tied to the direction of the respirationinduced motion of the heart, with attenuation correction artifacts positioned along this direction of motion.

The percentage differences between phase-matched and end-expiration corrections were larger than those with endinspiration correction. Because of the asymmetry of the respiratory cycle, with expiration encompassing two thirds of the cycle, it was expected that the end-expiration CT image would be closer to a respiration-averaged CT image than would the end-inspiration CT image, thus generating less error. However, this was not the case. The cause of this disparity is seen in Figures 6 and 7, which show the PET heart position with respect to the $\mathrm{CT}$ image for an example dog. When the PET heart image was at end-inspiration and was corrected with its phase-matched CT image, the heart appeared in the liver on the end-expiration CT image. Because of the large volume of lung being "replaced" by the liver at that transaxial level of the heart, this effect will greatly overcorrect that region of the heart when endexpiration CT attenuation correction is applied. In other words, a horizontal line, drawn through the region of the PET heart but incorrectly placed in the CT liver, contains more attenuating material than is actually experienced by photons exiting that region of the heart. Conversely, when the PET data at end-expiration are superimposed on the end-inspiration CT image, a small portion of the heart is placed in the lung region of the CT image. This region of the heart is, therefore, undercorrected when end-inspiration CT attenuation correction is applied, because a section of the heart will be attenuation-corrected as though it were lung at that sagittal level of the heart. In other words, a vertical line, drawn through the region of the PET heart but incorrectly placed in CT lung, contains less attenuating material than is actually experienced by that region of the heart. On examining these 2 figures, one can see that the region in the liver with end-expiration $\mathrm{CT}$ appears larger than the region in the lung with end-inspiration CT. This, combined with differences in the attenuation correction factor between the regions, leads to larger errors when endexpiration CT is used for attenuation correction than when end-inspiration CT is used.

Our experimental condition was idealized, because the dogs were sedated and ventilated. Both measures resulted in a regular, consistent breathing pattern, which may not occur clinically. Having a fixed respiratory period greatly simplified the acquisition of respiration-averaged CT because inconsistencies would otherwise arise between CT slices. Thus, the question is raised of what happens if the respiration-averaged $\mathrm{CT}$ is obtained incorrectly (i.e., not obtained over exactly 1 respiratory cycle), such as might occur if a patient's breathing rate changed during 4D CT acquisition. This question was examined in Figure 5, which compares the phase-matched-CT-corrected image with images obtained with incorrect respiration-averaged CT. The largest maximum percentage difference was only $3 \% \pm 1 \%$.

FIGURE 7. Fusion of phase-matched CT attenuation-corrected PET image at end-expiration with $\mathrm{CT}$ image at endinspiration: transaxial slice $(A)$, coronal slice (B), and sagittal slice (C).
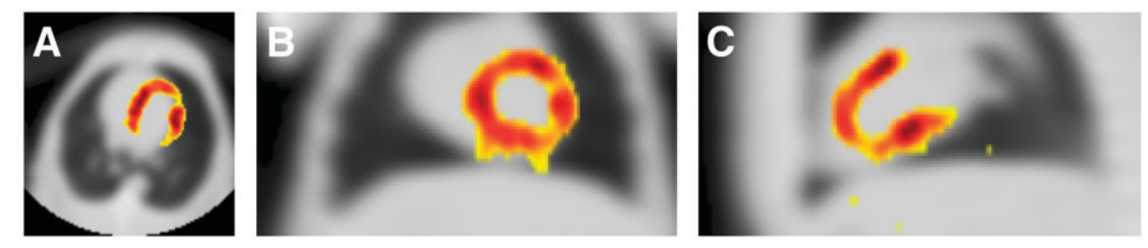
This value is twice the maximum difference of $1.6 \% \pm$ $0.7 \%$ that was seen when the correct respiration-averaged CT image was used for attenuation correction but still represents a large reduction over the error seen with singlephase CT corrections. This respiration-averaged CT attenuation correction is therefore quite robust with respect to small errors in the length of the respiratory cycle used in the averaging. Additionally, when the other 4 phase-shifted CT volumes were averaged and compared with the average of CT volumes used in the phase-matched correction of PET images, respiration-averaged CT was independent of phase shifts in the CT data, because all 4 phase-shifted corrections generated nearly indistinguishable results from the result seen in Figure 3B. This finding, by indicating that the specific phase of the cycle and duration of the scan do not greatly influence the accuracy of the correction, simplifies acquisition of respiration-averaged CT. Cine acquisitions of the patient can be obtained without the need for respiratory monitoring of the phase of acquisition.

Although the canine model is similar to humans, differences remain between the two, such as the magnitude of the attenuation correction factors and the breathing rate. The attenuation correction factor in humans can be 2 or 3 times larger than that in the dogs used in this experiment, and subsequently some of the differences in our data could potentially be even larger in humans. Also, the average breathing rate for humans is approximately 12-18 breaths per minute, producing a respiratory period of about $4 \mathrm{~s}$, compared with the $7.5 \mathrm{~s}$ used for the dogs in this study. The longer period was selected to minimize motion within each image of the 4D CT acquisition. Having a shorter respiratory period would increase the motion in each individual CT volume of the 4D CT acquisition. However, this effect should be small, because a cine mode acquisition using a 0.5 -s rotation speed is still fast enough to capture multiple phases of a 4-s respiratory cycle without producing significant respiration-induced image artifacts from inconsistencies in the projection data.

Another approach to obtaining a respiration-averaged CT scan of the patient is the use of slow-rotation CT (e.g., up to a 4-s rotation speed is available on our PET/CT scanner). This approach would not require gating, because the rotation speed and respiratory period would be similar. However, such an acquisition results in inconsistencies in the projection data because, during the course of the acquisition, projections are obtained from different phases of the respiratory cycle, leading to artifacts in the reconstructed images. Such drawbacks to slow-rotation CT have been shown in oncology studies (20).

One concern is the dose received by the patient during the multiple respiratory cycles required for 4D CT. However, accurate CT images can be obtained by reducing the $\mathrm{X}$-ray tube current from $80 \mathrm{~mA}$ (used in this study) to 10 $\mathrm{mA}$, and the dose to the patient would be reduced correspondingly (21). This approach would acquire an accurate respiration-averaged CT scan through 4D CT protocols, with only a small increase in dose relative to routine nonbreath-hold helical CT.

\section{CONCLUSION}

In respiration-ungated cardiac PET images from a live canine model, we compared 3 types of CT attenuation correction: CT of a single respiratory phase (either at end-inspiration, mid-cycle, or end-expiration), respiration-averaged CT, and phase-matched CT. Single-phase correction led to a maximum error of $11 \% \pm 4 \%$, when compared with phase-matched correction. This error was greatly reduced to $1.6 \% \pm 0.7 \%$ through the use of CT averaged over an entire respiratory cycle, as can be obtained with the 4D CT acquisition described by Pan et al. (9).

\section{ACKNOWLEDGMENTS}

We thank Jane Sykes, Lela Dorrington, Jennifer Hadway, Dominique Ouimet, Lynn Keenliside, and Benoit Lewden for their invaluable technical assistance with this research. This work was supported by the Heart and Stroke Foundation of Ontario (grant NA 5292 and a Masters award), the University of Western Ontario, the Ontario Research and Development Challenge Fund, the Lawson Health Research Institute, and GE Healthcare.

\section{REFERENCES}

1. Causes of Death 2002 (report 84-208-XIE). Ottawa, ON, Canada: Statistics Canada, Vital and Cancer Statistics Section; 2004.

2. Beaglehole R, Irwin A, Prentice T. The World Health Report 2004: Changing History. Geneva, Switzerland: World Health Organization; 2004.

3. Segall G. Assessment of myocardial viability by positron emission tomography. Nucl Med Commun. 2002;23:323-330.

4. Saab G, deKemp RA, Ukkonen H, et al. Gated fluorine 18 fluorodeoxyglucose positron emission tomography: determination of global and regional left ventricular function and myocardial tissue characterization. J Nucl Cardiol. 2003;10:297-303.

5. Zaidi H, Hasegawa B. Determination of the attenuation map in emission tomography. J Nucl Med. 2003;44:291-315.

6. Livieratos L, Stegger L, Bloomfield PM, Schafers K, Bailey DL, Camici PG. Rigid-body transformation of list-mode projection data for respiratory motion correction in cardiac PET. Phys Med Biol. 2005;50:3313-3322.

7. Ter-Pogossian MM, Bergmann SR, Sobel BE. Influence of cardiac and respiratory motion on tomographic reconstructions of the heart: implications for quantitative nuclear cardiology. J Comput Assist Tomogr. 1982;6:1148-1155.

8. Kinahan P, Hasegawa B, Beyer T. X-ray based attenuation correction for positron emission tomography/computed tomography scanners. Semin Nucl Med. 2003; 33:166-179.

9. Pan T, Lee T, Rietzel E, Chen G. 4D-CT imaging of a volume influenced by respiratory motion on multi-slice CT. Med Phys. 2004;31:333-340.

10. Fitzpatrick GM, Wells RG. Simulation study of respiratory-induced errors in cardiac position emission tomography/computed tomography. Med Phys. 2006;33:2888-2895.

11. Goerres GW, Burger C, Kamel E, et al. Respiration-induced attenuation artifact at PET/CT: technical considerations. Radiology. 2003;226:906-910.

12. Nehmeh SA, Erdi YE, Pan T, et al. Four-dimensional (4D) PET/CT imaging of the thorax. Med Phys. 2004;31:3179-3186.

13. Livieratos L, Rajappan K, Stegger L, Schafers K, Bailey DL, Camici PG. Respiratory gating of cardiac PET data in list-mode acquisition. Eur J Nucl Med Mol Imaging. 2006;33:584-588.

14. Chin B, Nakamoto Y, Kraitchman D, et al. PET-CT evaluation of 2-deoxy-2$\left[{ }^{18} \mathrm{~F}\right]$ fluoro-D-glucose myocardial uptake: effect of respiratory motion. Mol Imaging Biol. 2003;5:57-64. 
15. Le Meunier L, Maarss-Moreno R, Carrasquillo J, et al. PET/CT imaging: effect of respiratory motion on ${ }^{18} \mathrm{FDG}$ myocardial uptake [abstract]. J Nucl Med. 2005;46(suppl):163P.

16. Wassenaar R, Wells RG, deKemp R. Clinical evaluation of CT versus Ge-68 attenuation correction in cardiac PET imaging [abstract]. J Nucl Med. 2004;45(suppl):118P.

17. Pan T, Mawlawi O, Nehmeh SA, et al. Attenuation correction of PET images with respiration-averaged CT images in PET/CT. J Nucl Med. 2005;46:1481-1487.

18. Loghin C, Sdringola S, Gould KL. Common artifacts in PET myocardial perfusion images due to attenuation-emission misregistration: clinical significance, causes, and solutions. J Nucl Med. 2004;45:1029-1039.
19. Klein GJ, Reutter BW, Ho MH, Reed JH, Huesman RH. Real-time system for respiratory-cardiac gating in positron tomography. IEEE Trans Nucl Sci. 1998;45: 2139-2143.

20. Mori S, Kanematsu N, Mizuno H, Sunaoka M, Endo M. Physical evaluation of CT scan methods for radiation therapy planning: comparison of fast, slow and gating scan using the 256-detector row CT scanner. Phys Med Biol. 2006;51: 587-600.

21. Koepfli P, Hany TF, Wyss CA, et al. CT attenuation correction of myocardial perfusion quantification using PET/CT hybrid scanner. J Nucl Med. 2004;45: $537-542$. 